\title{
RHEED digital image analysis system for in-situ growth rate and alloy composition measurements of GaAs-based nanostructures
}

\author{
H. Sghaier, L. Bouzaiene, L. Sfaxi, H. Maaref \\ Laboratoire de Physique des Semiconducteurs et des composants Electroniques, Faculté des Sciences de Monastir \\ Avenue de l'Environnement, 5000 Monastir (Tunisia) \\ Phone: 2163 500280,fax: 2163 500278,E-mail:H.Sghaier@enim.rnu.tn
}

\begin{abstract}
Monitoring the intensity of the reflected spot in a RHEED image is the most important method used to control the growth of semiconductors in MBE. The accurate control of both layer thickness and alloy composition is particularly important for the growth of high quality heterostructures. Indeed, under such conditions, extremely uniform and high quality epitaxial devices become possible. RHEED intensity oscillation can be used as accurate, quick and direct measure of the growth rates and allow composition as well. Although analog signal could be obtained by using an optical fiber associated to a photo-detector PM tube and a plotter, this method is troublesome and limited. In some application, the availability of the intensity signal as a digital voltage is useful to realize further advanced analysis, and achieve feedback between growth dynamic and the external parameters, such as the cells temperatures and the synchronization of the shutters. In this paper we describe the advantages gained with the upgrade of our analog system into a digital package using CCD camera, frame grabber and a home made software. Its main purpose is to track RHEED intensity changes and measures the rate of oscillation. A state-of-the-art RHEED digital image analysis system gives us the necessary tools to gain insight into the thin film growth process and optimize material quality.
\end{abstract}

Keywords: electron diffraction, gallium arsenide, nanostructure.

Paper received 13.02.04; accepted for publication 17.06.04.

\section{Introduction}

Molecular beam epitaxy (MBE) is a sophisticated technique used in the growth of semiconductor layers, structures and devices. It is a technique that is of immense importance to both the industrial and academic sectors. MBE allows the controlled growth of semiconductor layers with monolayer (ML) precision [1]. A simple schematic of a typical MBE system is shown in Fig. 1. The layers are grown under ultra high vacuum conditions. Knudsen cells are used to deposit the various materials ( $\mathrm{Ga}, \mathrm{As}, \mathrm{In}, \mathrm{Al} \mathrm{Si})$ onto a heated substrate, which is rotated during growth to maximize growth homogeneity. Shutters are used to switch the molecular beams on and off and an ion gauge monitors the pressure. A very important in situ diagnostic tool used to monitor the film quality during growth is reflection high-energy electron diffraction (RHEED).

A wealth of fascinating physics has arisen from the ability to produce high quality semiconductor heterostructures using MBE. For example, the band gap of the AlGaAs compound varies with the relative concentrations of $\mathrm{Al}$ and $\mathrm{Ga}$ concentrations, which are directly controllable in MBE. The ability to both control the band gap of a material via changes in its composition (band gap engineering) and to epitaxially grow one layer of semiconductor on another revolutionized semiconductor fabrication processes and has led to the development of numerous important electronic and optoelectronic devices.

The accurate measurement of both alloy composition and growth rate is a key issue in order to obtain perfect devices, which exhibit interesting optoelectronic performance [2]. Monitoring RHEED pattern was traditionally done with optical fiber associated to a photomultiplier tube and a plotter. The method is simple but cumbersome to use; selecting the position of the spot is a time consuming and often frustrating job. Furthermore, the process of measurement done on the plotted curves is source of large uncertainty. For all the above mentioned limitations, a new digital RHEED package has been developed in our laboratory which has all features needed for the manufacturing of almost perfect devices. 


\section{H. Sghaier et al.: RHEED digital image analysis system for in-situ growth ...}

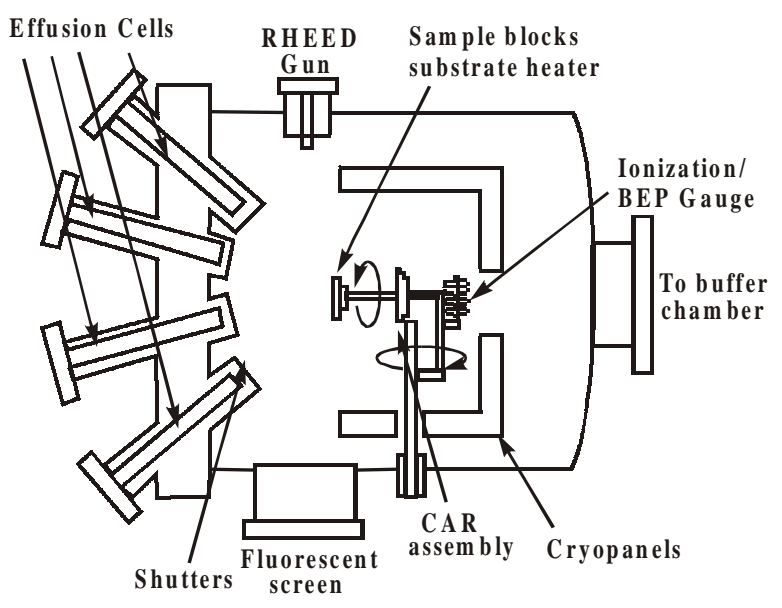

Fig. 1. Layout of a typical MBE system growth chamber

\section{RHEED signal detection and analysis}

One of the most useful tools for in-situ monitoring of the growth is reflection high-energy electron diffraction (RHEED). It can be used to calibrate growth rate, observe removal of oxides from the surface, calibrate surface temperature, monitor arrangement of the surface atoms, give feedback on surface morphology, and provide information about growth kinetics.

The layout of the RHEED system is shown in Fig. 2. The RHEED gun emits $12 \mathrm{keV}$, electrons, which strike the surface of at shallow angle (1-2 degrees), making it sensitive probe of the semiconductor surface. Electrons reflect from the surface and strike a phosphor screen forming a pattern consisting of specular reflection and a diffraction pattern, which is indicative of the surface crystallography. A camera monitors the screen and can record instantaneous pictures and measure the intensity of a given area of interest as a function of time. When growth is initiated on a smooth GaAs surface, the intensity of the RHEED pattern starts to oscillate. The oscillation frequency corresponds to the monolayer growth rate [3], when a monolayer is the thickness of one full layer of Ga and one full layer of As atoms. The oscillations can be explained by a layer by layer growth mode as demonstrated in Fig. 3. When a layer starts it is smooth and the specular spot is bright, but as the layer nucleates, islands form on the surface, and the specular spot dims. As the layer finishes, the islands coalesce into a flat layer, and the specular spot intensity increases.

The RHEED Digital Image Analysis System combines hardware and software into a package, which analyzes RHEED images in real time. Its main purpose is to track RHEED intensity changes and measures the rate of oscillation. It also has image analysis capabilities such as image capture and storage, surface plotting and profile analysis.

The detection is performed by a monochrome high sensitivity CCD camera looking at the RHEED screen. The camera is equipped with superior quality $16 \mathrm{~mm}$ fo-
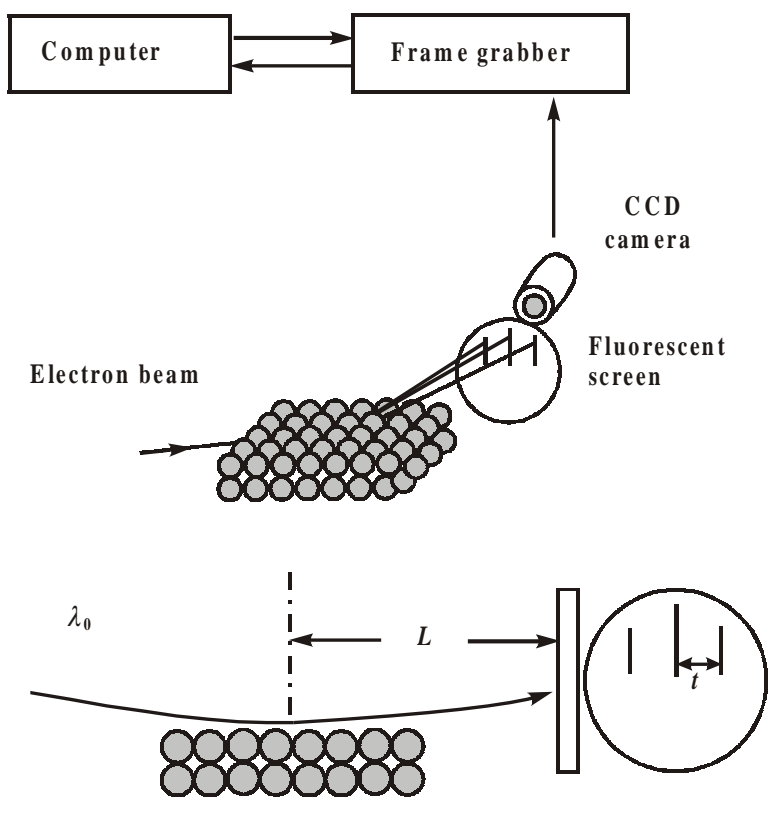

Fig. 2. Diagram of RHEED package

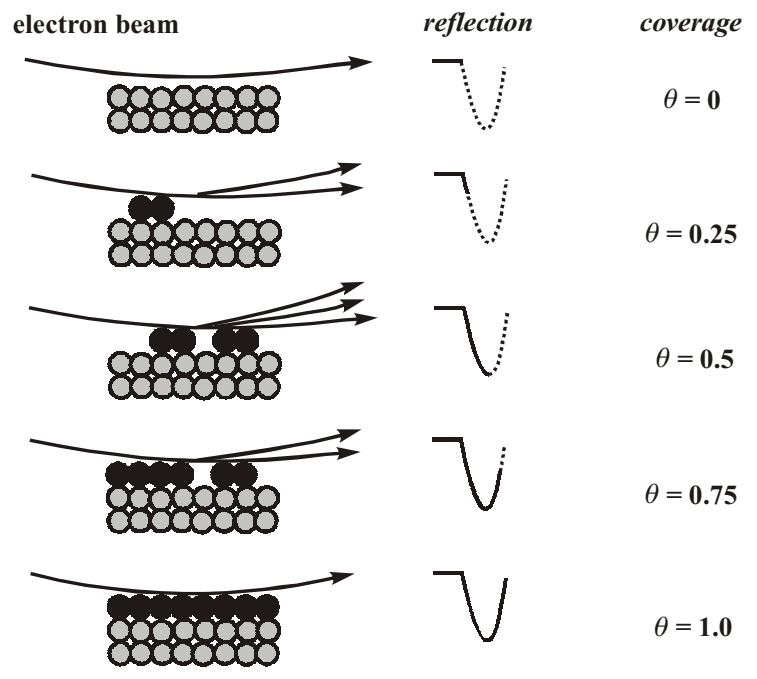

Fig. 3. Illustration of mechanism for RHEED specular spot oscillation during a growth of a monolayer

cal length lens. The high-speed shutter function and asynchronous random trigger function allow the camera to capture high quality images of fast moving objects making it suitable for applications such as dynamic RHEED acquisition during high speed substrate rotation. The mode of operation may be easily configured though a set of switches located on the rear panel. In particular, the automatic gain control should be switched off, since we 


\section{H. Sghaier et al.: RHEED digital image analysis system for in-situ growth ...}

are tracking light fluctuation over time. The camera continuously outputs images at a fixed frame rate, 30 frames per second in our case.

The output of the camera is an analog video signal, where timing signals are combined with video to form a composite video signal. The camera is interfaced to a computer via a PCI frame grabber that supports capture from the camera and provides real-time image transfer to the computer. Matrox Meteor-II digitizer has been adopted for its high performances, acquisition flexibility and low cost.

The images provided by the camera/digitizer combination are important source of information for interpretation and analysis. Software is needed to manage the acquisition and to analyze images. Numerous commercial software are available but none of them could fully meet our requirements. Thus, we have decided to build our own windows application using Delphi developing language.

The software being developed is a true 32-bit application that runs under windows 98 . It has an intuitive interface and includes a high-level programming library with an extensive set of optimized functions for image capture, display, analysis and archiving. The software receives its input from the frame-grabber and can measure and display changes in intensity that are far below what the unaided human eye can discern.
Intensity tracking and oscillation measurements are the main features of this software. To begin, a spot is defined on a live video RHEED image. Live image manipulation is possible, and on-screen controls are available for adjusting brightness, contrast and gain during live image capture. The digitization process divides an image into a two-dimensional grid of pixels. Each pixel has a value that corresponds to the intensity at that location in the image.

Not only can the software acquire a single image but it can also capture sequential images into PC memory or onto disk. Sequences are set of sequential frames that can be grabbed from a camera at a programmable sampling rate. The sampling period may be selected as low as $50 \mathrm{~ms}$. Once the sequence is grabbed, it may be analyzed and saved to disk in AVI format for further offline processing. Sequence control consists of a timer that indicates start, interval and stop times. Timing information is recorded and accessible separately from the images.

During playback the entire sequence can be viewed in a single pass or as a continuous loop. Image sequences can also be viewed as thumbnails of individual images. Processing is performed on a rectangular region of interest (ROI) of each single image (Fig. 4). For each frame,

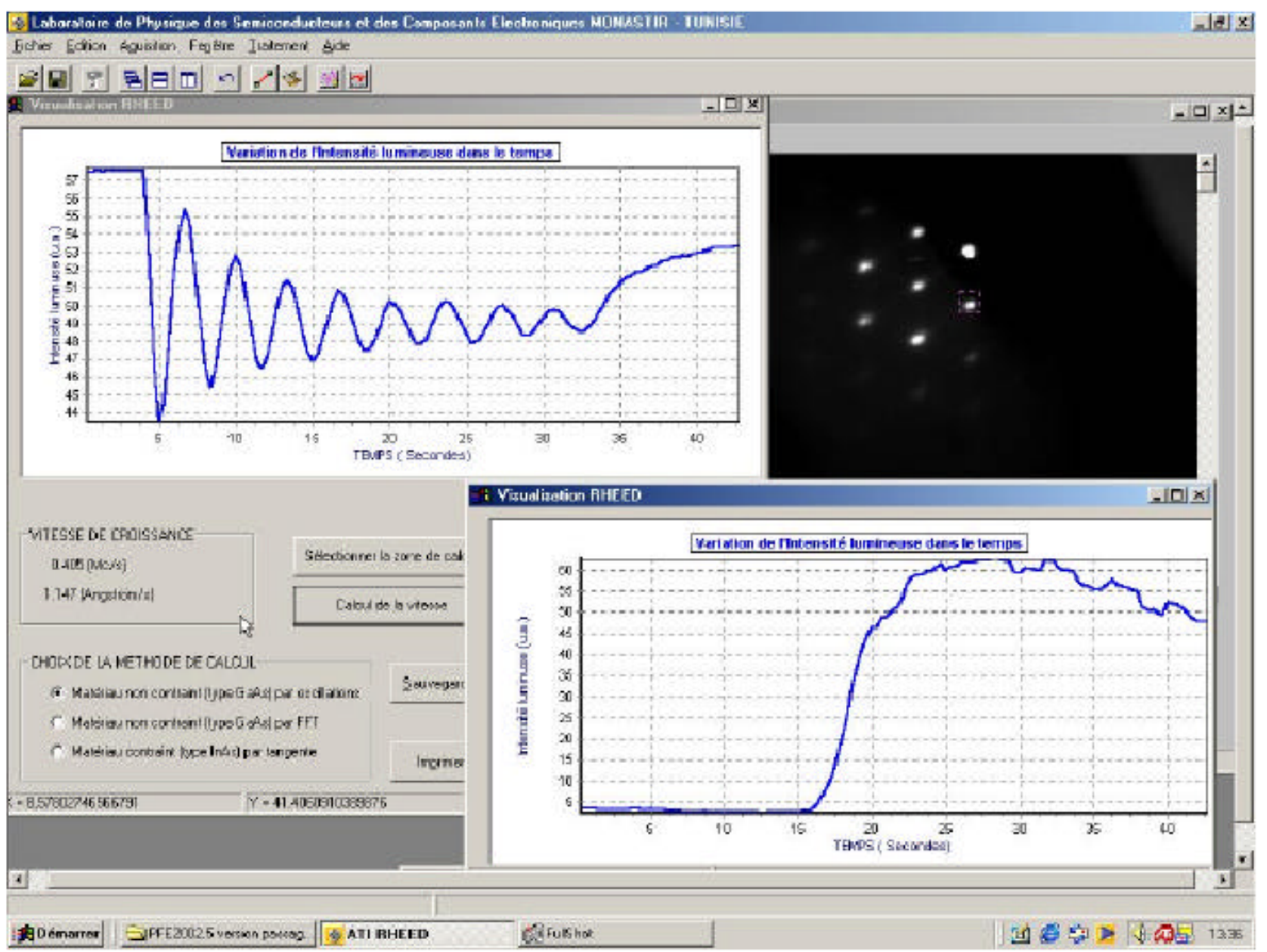

Fig. 4. Example of screens captured from the computer during actual MBE growth runs. 


\section{H. Sghaier et al.: RHEED digital image analysis system for in-situ growth ...}

the average pixel intensity of the selected ROI is calculated. A strip chart of intensity is plotted on the screen, the horizontal axis represents the timing of the image being processed, while the vertical axis represents the average pixel intensity. This data can be stored to disk, be printed out and have Fast Fourier Transform (FFT) analysis performed on it. FFT is used to determine automatically the growth rate by deriving the power spectrum of the intensity data. This spectrum is displayed on the screen, and the main frequency is calculated and displayed.

\section{Experimental}

The growth and RHEED studies were carried out in a RIBER32 MBE system equipped with conventional solid source effusion cells and a $12 \mathrm{keV}$ RHEED gun. Ga, Al, In and As elements were evaporated using effusion cells. Singular (001) GaAs substrates were used. Two different types of structures have been studied: AlGaAs, InGaAs quantum wells, and InGaAs/GaAs quantum dots.

The samples are outgassed in the buffer chamber at $450^{\circ} \mathrm{C}$ before they are moved into the growth chamber. After the samples are loaded into the chamber and moved to face the sources, the RHEED screen will show a haze, which is indicative of the amorphous nature of the protective oxide layer. The temperature is ramped up, with As overpressure, until a diffraction pattern appears, showing that the oxide has desorbed on the surface. The temperature of the substrate is monitored using a thermocouple in direct contact with the molybdenum block. We use the deoxidation temperature as a reference point for 600 ${ }^{\circ} \mathrm{C}$ [4]. After RHEED shows that the oxide is gone, the sample temperature is increased about $50^{\circ} \mathrm{C}$ for few minutes to ensure that all traces of oxide are removed.

The growth rates and alloy compositions were measured using the newly developed RHEED package. The variation of the In and $\mathrm{Al}$ compositions was accomplished by increasing the temperature of the In and Al sources. The composition is determined from the growth rate as obtained from the period intensity oscillations, utilizing that the sticking coefficient of both $\mathrm{Ga}$ and In is unity under As rich conditions, the GaAs growth rate serves as a reference. The RHEED pattern was recorded using extremely sensitive CCD camera coupled to a frame grabber and a computer.

\section{Results and discussions}

RHEED intensity behavior during the growth of $\mathrm{Al}_{\mathrm{X}} \mathrm{Ga}_{1-\mathrm{x}}$ As on GaAs.

One of the most important discoveries in RHEED applications is the finding of periodic RHEED intensity oscillation, which corresponds to the monolayer deposition time [5]. In Fig. 5 a typical RHEED image is shown, displaying a diffraction pattern. When a layer starts it is smooth and the specular spot is bright, but as the layer nucleates, islands form on the surface, and the specular spot dims. The appearance of the RHEED diffraction pattern can be used to

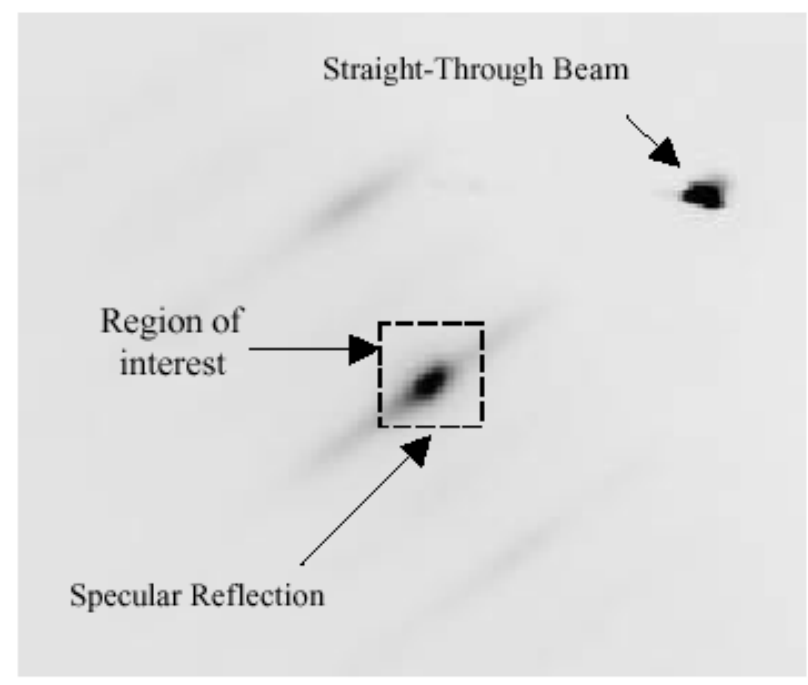

Fig. 6. Intensity of the RHEED specular spot during growth of GaAs and AlGaAs. The oscillations correspond to growth rate of $1 \mathrm{~A} / \mathrm{s}$ for $\mathrm{GaAs}$, and $1.8 \mathrm{~A} / \mathrm{s}$ for AlGaAs. Aluminum concentration $\mathrm{X}_{\mathrm{Al}}$ may be deduced by computing $\mathrm{V}_{\mathrm{GaAs}}$ and $\mathrm{V}_{\mathrm{AlGaAs}} \cdot \mathrm{X}_{\mathrm{Al}}=$ $=44 \%$ in our example.

provide qualitative feedback on the surface morphology. If the surface is smooth, then RHEED diffraction patterns appears streaky, similar to what is seen in Fig. 5. If the sample is rough, then the streaks are spottier. RHEED oscillation for GaAs and AlAs growth starting on a GaAs surface is shown in Fig. 6. At the start of growth, the intensity is greatest since the layers are nearly atomically smooth. The magnitude of the RHEED oscillations damps because as the growth progresses, islands nucleate before the previous layer is finished.

RHEED imaging is also used to determine the composition of AlGaAs layers. Before each growth run, GaAs

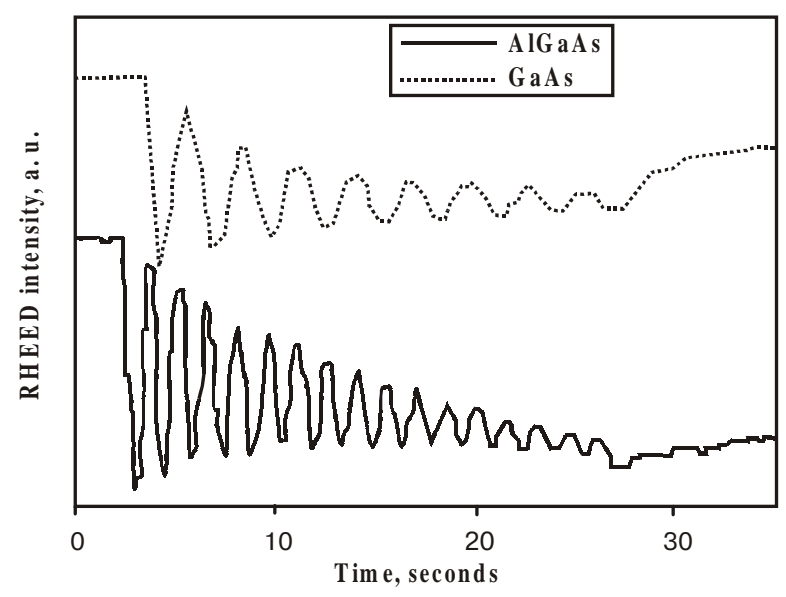

Fig. 5. Example of RHEED pattern obtained during GaAs growth. The software will take an average of the pixels in the selected region of interest (ROI), and plot use that data for the rest of calculations. 


\section{H. Sghaier et al.: RHEED digital image analysis system for in-situ growth ...}

and $\mathrm{AlGaAs}$ growth rates were measured separately using the monolayer oscillation in the intensity of the zeroorder beam in the RHEED pattern [6-7]. The Al mole fraction $X_{\mathrm{Al}}$ was calculated by dividing the difference between the AlGaAs and GaAs growth rates by the $\mathrm{AlGaAs}$ growth rate [7]:

$$
X_{\mathrm{Al}}(\%)=\text { Erreur! }
$$

RHEED intensity behavior during the growth of $\mathrm{In}_{\mathrm{x}} \mathrm{Ga}_{1-\mathrm{x}}$ As on GaAs

Because the layers must be lattice-matched, the growth rates must be calibrated accurately to achieve high quality material. The same technique used to calibrate AlGaAs material can be used for a rough estimation of In growth rate, but is not reliable. In fact, because of the low In growth rate, the oscillations will be poor and therefore will have a large uncertainty. Alternatively, a more indirect method must be used [8]. During the InAs deposition, RHEED observations have shown that at certain thickness of InAs, the RHEED pattern was rapidly but continuously transformed into a spotty pattern with weak streaks. Subsequently the spots gradually became more intense, which accompanied the disappearance of the streaks (Fig. 7). 2D-3D transition corresponds to the formation of $1.7 \mathrm{ML}$ of InAs. Fig. 8 shows recorded time dependence of Bragg reflection during the growth of InAs on GaAs. When the 2D to 3D growth mode transition occurs, a fast increase of the intensity is observed at the Bragg spot. This is a direct consequence of the increase of surface roughness associated with the formation of 3D islands.

\section{Reproducibility of the growth rate}

The features of MBE growth are layer thickness control-

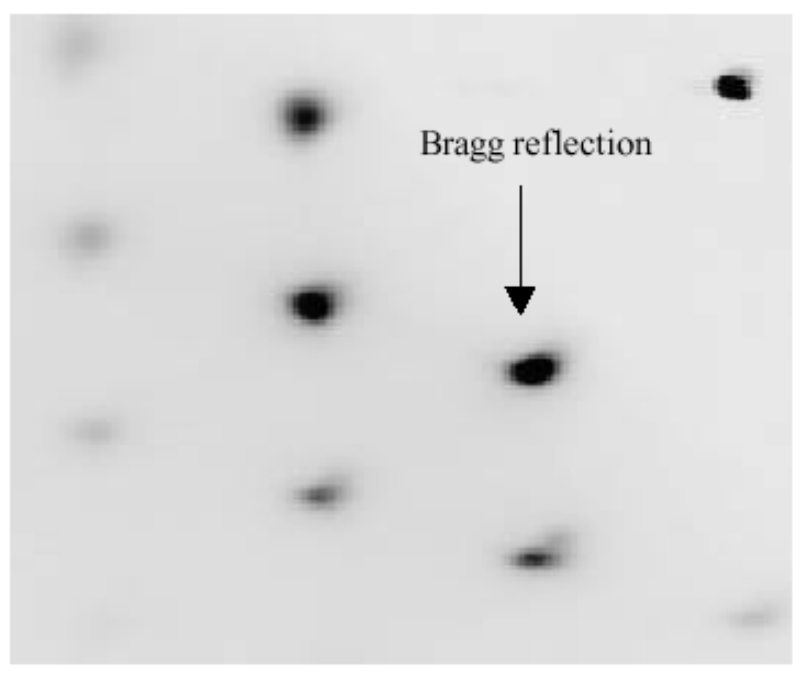

Fig. 7. Example of RHEED pattern obtained during growth of InAs. The spotty image is indicative of $2 \mathrm{D}-3 \mathrm{D}$ transition.

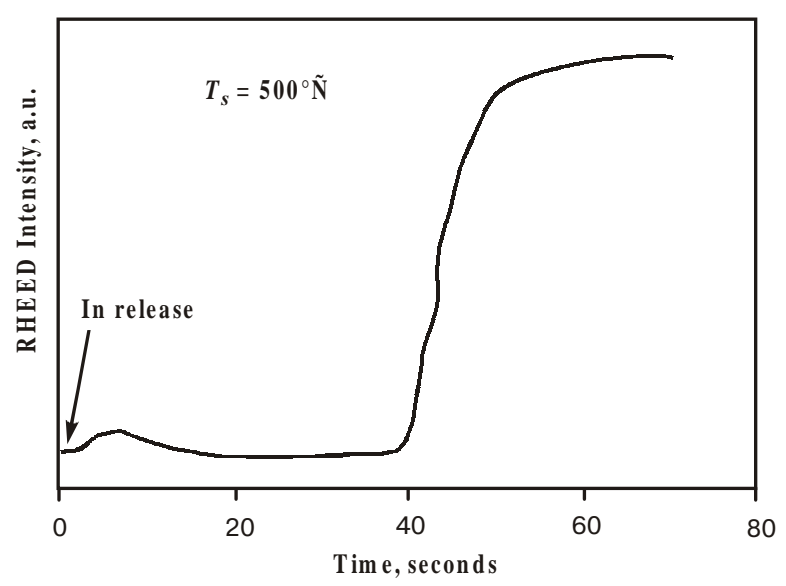

Fig. 8. Intensity variation of Bragg reflection recorded during the growth of InAs at $500^{\circ} \mathrm{C}$. The sharp 2D-3D growth mode transition corresponds to the build-up of $1.7 \mathrm{ML}$.

lability and sharp growth interface. Since our MBE system is mainly used for research, only few runs per month are scheduled. Therefore, by using the calibration curves (Fig. 9), we could accurately control the growing layer thickness throughout a long period at good reproducibility. The growth rate as a function of temperature was found to be:

$$
\begin{aligned}
& \log \left(\mathrm{V}_{\text {GaAs }}\right)=8.410^{-3} T-7.4, \\
& \log \left(\mathrm{V}_{\text {InAs }}\right)=13.210^{-3} \mathrm{~T}-10.1
\end{aligned}
$$

Nevertheless, calibration curves do shift over time as the source materials are depleted from the crucibles. As the source material of effusion cells decreases, the beam intensity decreases as well. To obtain a material beam, at con-

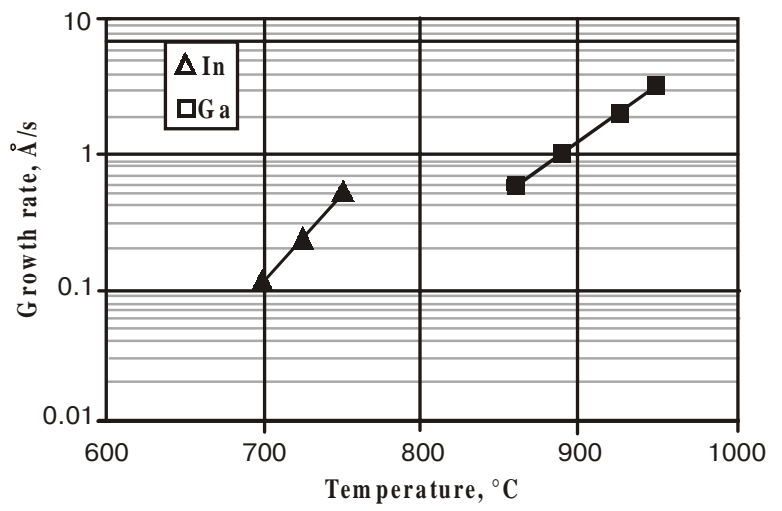

Fig. 9. Variation of the growth rate of In and $\mathrm{Ga}$ elements as a function of the evaporation cells temperature. The solid curves are fits of experimental data. 


\section{H. Sghaier et al.: RHEED digital image analysis system for in-situ growth ...}

stant intensity, effusion cell temperature must be increased at a specified rate.

\section{Low-dimensional structures}

In recent years, much research has been focused on selforganized ensembles of quantum dots. Such quantum dots are quasi-zero-dimensional objects (with size of 5$20 \mathrm{~nm}$ ), which form during hetero-epitaxial growth due to the mismatch of the lattice parameters of the growth material and the substrate.

Semiconductor quantum dots have already been utilized in electronic and optoelectronic devices, such as infrared photodetectors [9], semiconductor lasers [10-13], light emitting diodes [14-16], single electron transistors [17], and more recently single photon emitters [18]. QDs may also allow one to extend the emission wavelength of GaAs-based devices to the telecommunication wavelength range of 1.3-1.55 $\mu \mathrm{m}$ [19].

The formation of quantum dots is driven by the strain during epitaxy growth of InGaAs on a GaAs substrate as the deposited layer exceeds a critical thickness. Correspondingly the growth mode switches from a two dimensional growth to a three dimensional growth. The strain is relieved elastically without introduction of crystal defects. The dot size, areal density, and optical properties depend on the growth parameters, such as growth rate and group III over group V ratio [20-21]. In particular, the emission wavelength of InAs QDs can be tuned by varying the growth conditions, thus covering the important telecommunication wavelength range. Therefore, growth parameters should be accurately tuned in order to get the most perfect devices.

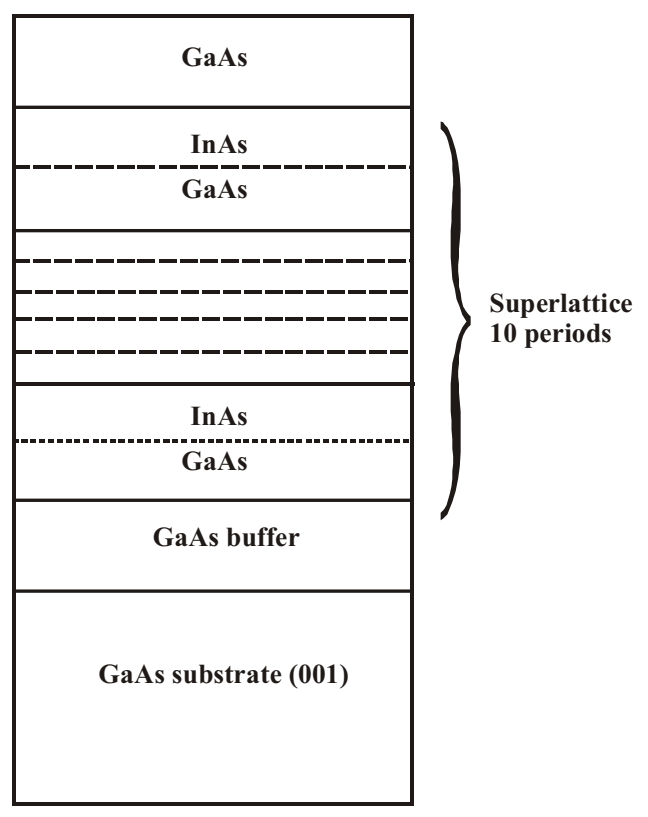

Fig. 10. InGaAs multiquantum wells test structure

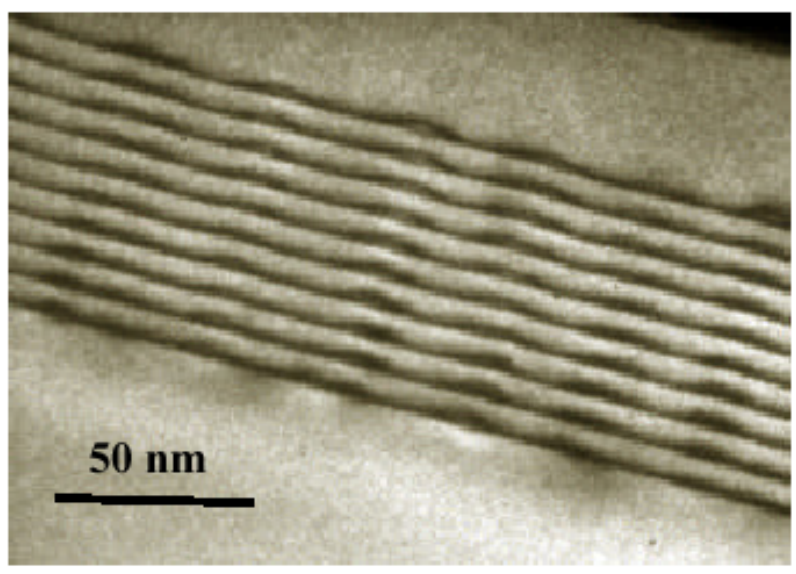

Fig. 11. TEM cross-sectional image for a sample with 10 numbers of layers with InAs QDs separated by $30 \mathrm{ML} \mathrm{GaAs} \mathrm{spacer.}$

Once quantum dots or wires form on the surface we must be able to examine and observe them with high resolution if we are to probe and understand their growth. Using RHEED data we can quickly, and with $0.1 \mathrm{ML}$ accuracy, identify the critical thickness for a surface transition from $2 \mathrm{D}$ layers to $3 \mathrm{D}$ islands. This is because $3 \mathrm{D}$ islands are distinguished by a $3 \mathrm{D}$ diffraction pattern that is strikingly different than the typical 2D diffraction pattern. Moreover, RHEED may be combined with photoluminescence (PL) measurements to study Indium surface segregation during molecular beam epitaxy of InGaAs on $(h, 1,1)$ GaAs [22].

Deposition of short period superlattices of III-V alloys have been shown to result in lateral composition modulation witch produces vertically coupled QD structures under optimized growth conditions [23]. A couple of short-period supperlattices structures were grown by $\mathrm{MBE}$ at a temperature of $500^{\circ} \mathrm{C}$ on semi-insulating (001) GaAs substrate. A schematic diagram of the structure is given in Fig. 10. Following a 50-nm-thick GaAs buffer layer, InAs QDs were formed under a repeated growth sequence of $2.4 \mathrm{ML}$ InAs followed by $30 \mathrm{ML}$ GaAs spacer. Growth rate was calibrated by newly developed RHEED package.

RHEED was also used to monitor the sample surface during the growth of InAs QDs formation. It is interesting to point out that $2 \mathrm{D}$ to $3 \mathrm{D}$ growth mode transition according to the RHEED pattern occurred at the same amount of deposited InAs layer, approximately 1.7 ML. The resulting lateral composition modulation was characterized by crosssectional transmission electron diffraction (TEM). In Fig. 11, TEM cross-sectional image for multilayer sample with 10 QDs layers separated by GaAs 30 ML thickness spacer is shown. A very strong vertical correlation is clearly seen. These results show that we have successfully produced quantum well wires by deposition of GaAs/InAs short period superlattices. Nevertheless, further adjustments of both GaAs spacer and InAs thickness are necessary to improve vertical QDs correlation. 


\section{H. Sghaier et al.: RHEED digital image analysis system for in-situ growth ...}

\section{Conclusions}

In summary, we have developed a RHEED digital image analysis system that combines hardware and software into a package, which analyses RHEED images in real time. The system uses CCD camera, PC framegrabber and a home made advanced software. Its main purpose is to track RHEED intensity changes and measures the rate of oscillation. It also has image analysis capabilities such as image capture and storage, filtering and profile analysis. The developed package gives us the necessary tools to gain insight into the thin film growth process and optimize material quality.

A couple of samples have been grown by MBE to check the ability of our system to accurately calibrate growth rate, to determine alloy composition, and to probe 2D to 3D transition growth mode during QDs formation as well. PL and TEM results clearly reveal that we can rely on our developed RHEED instrument.

Actually, our system is being used mainly for growth rates evaluation, alloy's composition measurements, and surface quality monitoring. In the near future we are planing to enhance it by connecting the RHEED package to the already automated cell's shutters [24]. Therefore, by using the RHEED specular spot oscillation to adequately feedback the opening and closing of the shutters, it is possible to build an almost perfect device.

Quantum dots are promising structures for the future. Our instrument allows a more controlled formation of quantum dots, which leads to better reproducibility and superior optical quality. Therefore, it may be used in conjunction with others techniques, such as PL, TEM, and AFM, for a systematic investigation that considers growth conditions for promising InAs/GaAs QDs and quantum wires based devices.

\section{References}

1. A.Y. Cho // Journal of Crystal growth 150(1995) p. 1.

2. J.C. Garcia, C.Neri, J.Massies // Journal of crystal growth, 98, p. 511 (1989) .

3. J. Zhang, A.K.Lees, A.g.Taylor, D.Raisbeck, N.Shukla, J.M. Fernandez, B.A. Joyce, M.E. Pemble // Journal of Crystal growth, 164, p. 40 (1996).

4. B. Etienne, E. Paris // Journal of Physics, p. 2049 (1987).

5. J.H Neave, B.A. Joyce, P.J. Dobson, N. Norton // Appl. Phys., A31, p. 1 (1983).

6. J.M. Van Hove, C.S Lent, P.R. Pukite, P.I. Cohen // J. Vac. Sci. Technol., B1, p. 741 (1983).

7. F.Turco, J. Massies, J.P.Contour // Rev. Phus. Appl., 22, p. 827 (1987).

8. A. Marti Ceshin // J. Massies. Journal of Crystal Growth, 114, p. 693-699 (1991).

9. H.C. Liu, M. Gao, J. McCaffrey, Z.R. Wasilewski, S.Fafard // Applied Physics Letters, 78(1), p. 79 (2001).

10. D.L. Huffaker, G. Park, Z. Zou, D.G. Deppe // Appl. Phys. Lett., 73, p. 2564 (1998).

11. A.E. Zhukov et al. // IEEE Photonics Technol. Lett., 11, p. 1345 (1999).

12. Y.M. Shernyakov et al. // Electron. Lett., 35, p. 898 (1999).

13. G.T. Liu, A. Stintz, H. Li, T.C. Newell, A.L. Gray, P.M. Varangis, K.J. Malloy, L.F. lester // IEEE J Quantum electron., 36, p. $1272(2000)$.

14. D.L. huffaker, D.G. Deppe // Appl. Phys. Lett., 73, p. 520 (1998).

15. A. Fiore, U. Oeserle, R.P. Stanley, M. Ilegems // IEEE Photonics Technol. Lett., 12, p. 1601 (2000).

16. J.X. Chen, U. Oesterle, A. Fiore, R.P. Stanley, M. Ilegems, T. Todaro // Appl. Phys. Lett., 79, p. 3681 (2001).

17. H. Drexler, D. Leonard, W. Hansen, J.P. Kotthaus, P.M. Petroff // Phys. Rev. Lett., 73, p. 2252 (1994).

18. V. Zwiller, H. Blom, L. Samuelson, G. Bjork // Appl. Phys. Lett., 78, p. 2476 (2001).

19. F. Ferdos, M. Sadeghi, Q.X. Zhao, S.M. Wang, A.Larsson / / Journal of Crystal growth, 227-228, p. 1140-1145(2001) .

20. S. Fafard,, Z.R. Wasilewski, M. Spanner // Applied Physics Letters, 75, p. 1866 (1999).

21. J.S. Kim, P.W. Yu, J.I. Lee, J.Su. Kim, S.G. Kim, J.Y. Leem, M. Jeon // Applied Physics Letters, 80, p. 25 (2002).

22. M. Ilg, K.H. Ploog. Phys. Rev. B 48, 15 (1993) [23] Q. Xie, A. Madhukar, P. Chen, N.P. Kobayashi // Phys.Rev.Lett, 75(13) p. 2542 (1995).

24. H. Sghaier, L. Sfaxi, H. Maaref. $11^{\text {th }}$ European Workshop on Molecular Beam Epitaxy (2001). 